\title{
HARPO, prototype of a gamma-ray polarimeter: Results of a polarised photon beam test between 1.7 and $74 \mathrm{MeV}$
}

\author{
David Attié* \\ IRFU, CEA, Université Paris-Saclay, F-91191 Gif-sur-Yvette, France \\ E-mail: david.attie@cea.fr
}

S. Amano ${ }^{a}$, P. Baron ${ }^{b}$, D. Bauduin ${ }^{b}$, D. Bernard ${ }^{c}$, P. Bruel $^{c}$, D. Calvet ${ }^{b}$, P. Colas $^{b}, \mathbf{S}$.

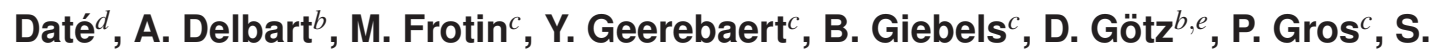
Hashimoto $^{b}$, D. Horan ${ }^{c}$, T. Kotaka ${ }^{b}$, M. Louzir ${ }^{c}$, F. Magniette $^{c}$, Y. Minamiyama ${ }^{b}$, S. Miyamoto $^{b}$, H. Ohkuma ${ }^{d}$, P. Poilleux ${ }^{c}$, I. Semeniouk ${ }^{c}$, P. Sizun ${ }^{b}$, A. Takemoto ${ }^{a}$, M. Yamaguchi $^{a}$, R. Yonamine ${ }^{b}$, S. Wang ${ }^{c}$

${ }^{a}$ LASTI, University of Hyôgo, Japan

${ }^{b}$ IRFU, CEA, Université Paris-Saclay, F-91191 Gif-sur-Yvette, France

${ }^{c}$ LLR, Ecole Polytechnique, CNRS/IN2P3, Palaiseau, France

d JASRI/Spring8, Japan

${ }^{e}$ Univ. Paris Diderot, AIM, Sorbonne Paris Cité, CEA, CNRS, F-91191 Gif-sur-Yvette, France

Access to the photon polarisation in the $1-100 \mathrm{MeV}$ energy range is a challenge for the next generation of space telescopes. The current telescopes in space are almost blind in this energy range, mainly due to the degradation of the angular resolution of e+e- pair and due to elastic scattering in the matter. Pair-conversion detector technologies as gaseous detectors are a promising alternative to the technologies based on tungsten-converter/thin-sensitive-layer stacks such as COS-B/EGRET/Fermi-LAT, firstly to improve the single-photon angular resolution and secondly for the polarisation information.

The use of a time projection chamber (TPC) as a target and a tracking detector will improve by up to one order of magnitude the single-photon angular resolution $\left(0.5^{\circ} @ 100 \mathrm{MeV}\right)$ with respect to the Fermi-LAT ( $5^{\circ} @ 100 \mathrm{MeV}$ ), and by up to a factor of three with respect to what can be expected for silicon detectors (1.0-1.5 @ $100 \mathrm{MeV})$. With such a good angular resolution, a TPC can close the sensitivity gap at the level of $10^{-6} \mathrm{MeV} / \mathrm{cm}^{2}$.s) between 3 and $300 \mathrm{MeV}$ despite having e lower sensitive mass. Furthermore, this good single-track angular resolution allows us to measure the linear polarisation fraction.

The HARPO (Hermetic ARgon POlarimeter) detector prototype that we built is a high pressure (0.5-4 bar) low pile-up and low-diffusion gas detector. We will present the results of its highstatistics characterisation in the 1.7-74 MeV fully-polarised and non-polarised gamma-ray beam provided by the BL01 line at NewSUBARU. The excellent value of the polarisation asymmetry dilution factor that we measured opens the possibility of having a polarimeter in space working in the $\mathrm{MeV}-\mathrm{GeV}$ energy range. In conclusion, we will present the design of a balloon-flight prototype ST3G (Self-Triggered Time projection chamber as a Gamma-ray Telescope) which is being developed. We will discuss its expected performance.

35th International Cosmic Ray Conference - ICRC2017 -

10-20 July, 2017

Bexco, Busan, Korea 


\section{Introduction}

Since decade the measurement of high energy photons (X-ray and $\gamma$-ray) polarisation is a challenge for each new experiment proposal. Indeed, the polarisation information from discrete sources will give access to the processes generating hard electromagnetic radiations allowing the discrimation between synchrotron self-Compton in leptonic model and proton synchroton in hadronic model [1]. Only hard X-ray and $\gamma$-ray photons are able to give a polarisation signature allowing to distinguish between the leptonic and hadronic radiation models. Depending on the energy of the $\gamma$ photon, the measurement of its polarisation will use one of the mechanisms as Compton scattering, electron-positron pair production or photodisintegration in strong resonance regions. Using a gaseous detector to produce electron-positron pair tracks can give access to the 1-100 $\mathrm{MeV}$ range with a better angular energy resolution $\left(0.5^{\circ} @ 100 \mathrm{MeV}\right)$ while measuring the linear polarisation fraction of the photons [2]. To demonstrate and validate this performance, a prototype of time projection chamber (TPC) was built and operated in $\gamma$-ray beam.

First, we will briefly describe the detector and the data taking in $\gamma$-ray beam using polarised and non-polarised photon at NewSubaru facility, then the data analysis and the results will be presented and finally we will discuss of the design of the trigger for a balloon flight prototype currently in progress.

\section{Prototype desciption and New Subaru photon beam campaign}

HARPO (Hermetic ARgon POlarimeter) is a demonstrator for a high angular resolution and high sensitive teslecope and polarimeter using the electron-position pair production. It consists of a 30-cm cubic vessel filled with high-pressure argon-based gas operating as a target and a tracking detector in TPC mode read out by micro-patterned technologies. The readout plane is composed of two times 288 strips of $1 \mathrm{~mm}$ pitch in the two perpendicular directions (X and Y) connected to electronic chain sampled at $33 \mathrm{MHz}$ allowing 3D point resolution of $\sim 1 \mathrm{~mm}^{3}$. The elecronics chain is composed of a T2K/TPC front-end card, a FEMINOS back-end card for each axis, both connected to a Trigger Clock Module for synchronisation and collection ([3]). The TPC was filled with a mixture of $5 \%$ of $\mathrm{iC}_{4} \mathrm{H}_{10}$ in argon and was operated at $220 \mathrm{~V} / \mathrm{cm}$ of electric field to drift the primary electrons to the readout plane composed by two GEM foils [4] and a bulk Micromegas $[5,6]$ on top of the strips. The detector, the electronic chain and its innovative trigger system called FAST (Fully Automated Switched Trigger) have been described in details in [7, 8, 9].

The beam test took place in November 2014 at the BL01 beam line of the Spring8/NewSUBARU synchroton which is operated by the Laboratory of Advanced Science and Technology for Industry (LASTI, University of Hyôgo, Japan). The photon are created using inverse Compton scattering by injecting the laser photons on the relativistic electrons from the synchroton. By changing the wave length of the laser and the energy of the electron beam, monochromatic $\gamma$-ray photons with an energy between $1.7 \mathrm{MeV}$ and $74.3 \mathrm{MeV}$ were produced with a rate more than $2 \mathrm{kHz}$. The polarization state of the photons coming from the laser are transferred to the $\gamma$-ray photons. The linear polarisation of the laser was measured to be above $99.9 \%$.

\footnotetext{
* Speaker.
} 
Half of the $60 \times 10^{6}$ events which were recorded, was $\gamma$ conversion event with a trigger rate of $50 \mathrm{~Hz}$ selected by the FAST trigger over the incoming background rate of about $10 \mathrm{kHz}$. Figure 1a shows how the data is extracted from the two front-end cards wich read both coordinates $\mathrm{X}(\mathrm{t})$ and $\mathrm{Y}(\mathrm{t})$. A typical event of $\gamma$-ray conversion by pair production, Compton scatterring and triplet are respectively shown on Figure 1b, 1c and 1d.

(a) Event in the TPC in $\mathrm{X}(\mathrm{t})$ and $\mathrm{Y}(\mathrm{t})$ readout.
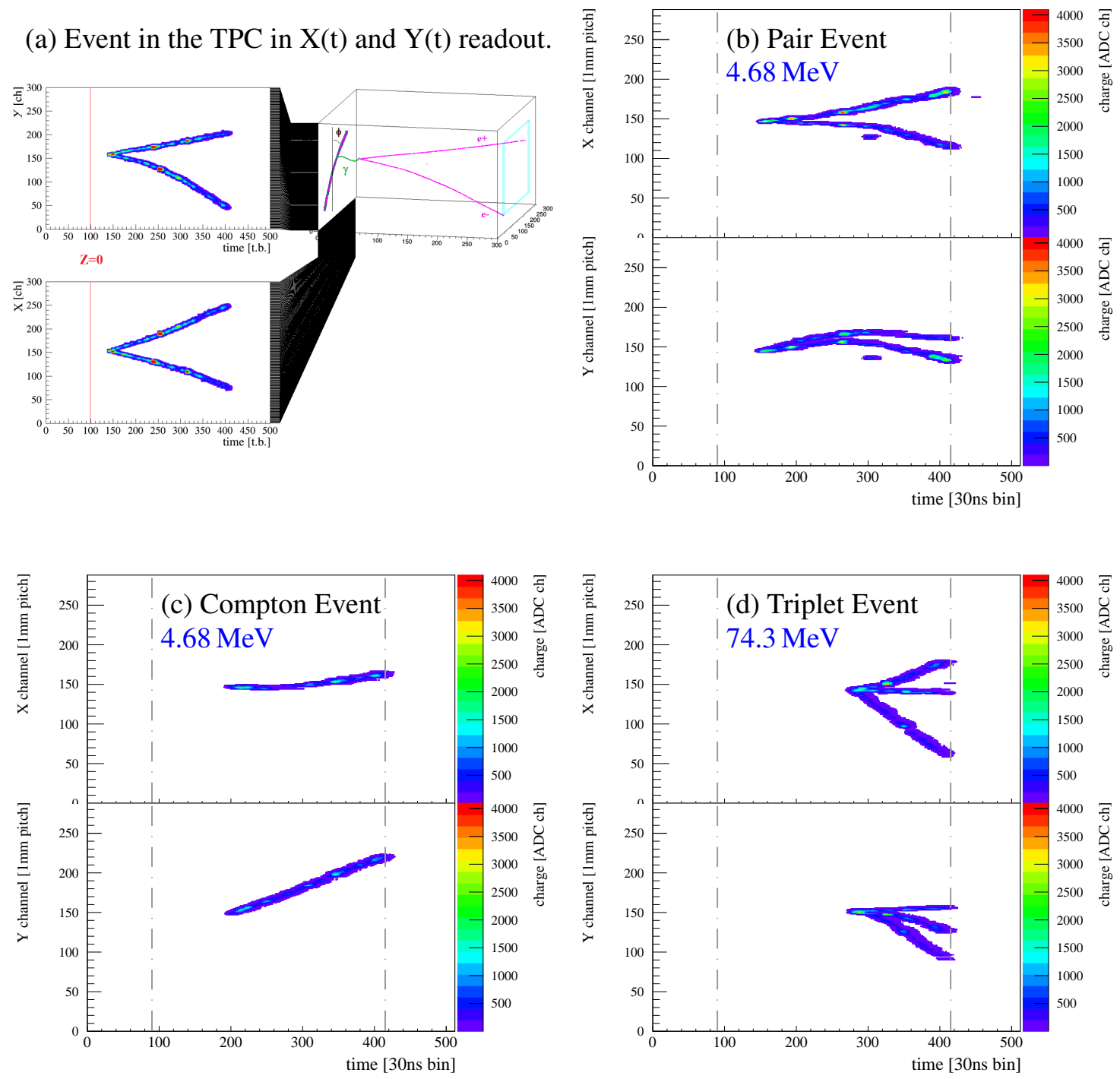

Figure 1: (a) Readout of an event using the TPC using $\mathrm{X}(\mathrm{t})$ and $\mathrm{Y}(\mathrm{t})$ strips. The three events are identified respectively as Compton scattering event at $4.68 \mathrm{MeV}(\mathrm{c})$, pair conversion event at 4.68 MeV (b), and triplet conversion event at 74,3 MeV (d).

\section{Data analysis and results}

The data analysis was suffering of a pre-amplifier saturation for tracks perpendicular to the 
readout plane (nominal configuration during the beam tests). So sometimes single tracks events could be misreconstructed as pair (see Sec. 3 of [10]). However, this saturation effect will be negligible in space or for a ballon flight while the tracks will be isotropic. The opening angle with a small opening angle is given by the Equation 3.1:

$$
\theta_{ \pm}=\arccos \left(\vec{u}_{\text {pair }} \cdot \vec{u}_{\gamma}\right)
$$

where $\vec{u}_{\gamma}$ is the beam direction and $\vec{u}_{\text {pair }}=\vec{u}_{+}+\vec{u}_{-}$the corresponding photon direction from the recontructed vertex. A vertex could have one track $\left(\theta_{ \pm}=0\right)$ or two tracks $\left(\theta_{ \pm}>0.05+\frac{0.5 \mathrm{MeV}}{E_{\gamma}}\right.$ in radian) depending on the photon energy $E_{\gamma}$.

A simulation framework have been developped in order to understand the data (Sec. 5 in [11]). The simulated data were also used to remove the systematic bias derived from the detector acceptance in measuring the polarisation. Our simulation was firstly validated with cosmic-rays and its parameters were calibrated with the beam test data. The photon direction is extracted by taking the bisector of the $\mathrm{e}^{+} / \mathrm{e}^{-}$momentum directions in the pair production process. The measured polarisation asymmetry $(A)$ and the distribution of the azimuthal angle $\phi_{ \pm}$are defined by the equation $1+A \cos 2\left(\phi_{ \pm}-\phi_{0}\right)$ where $\phi_{ \pm}=\frac{\phi_{+}+\phi_{-}}{2}$ (See figure 2$)$.

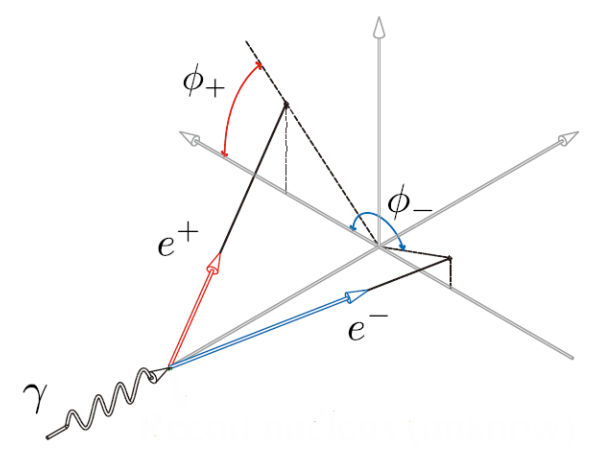

Figure 2: Angle definition of the $\gamma$-ray conversion into an electron-positron pair.

Due to large diffusion in the gas (no magnetic field), $\mathrm{X}(\mathrm{t})$ and $\mathrm{Y}(\mathrm{t})$ correlation and the important multiple scattering of the electrons and positrons make difficult the use a straight line finding algorithm (Hough transform) or more sophisticated tracking method as a Kalman filter. So, in order to identify the pair vertices, an original method has been developed. Then by fitting the vertices of the two projections of $\mathrm{X}(\mathrm{t})$ and $\mathrm{Y}(\mathrm{t})$, the plan $\mathrm{XY}$ and $\mathrm{XZ}$ are associated with each other to recover the full 3D description of the vertex. The differents steps are briefly desribed in the following subsections (for more details, see [12]).

\subsection{Search for 2D vertex}

A vertex is defined to be the starting point of two tracks where a photon is converted into a $\mathrm{e}^{+} \mathrm{e}^{-}$pair. So, the angle defined by the tracks should strictly inferior to $\pi$. The wertex finder consist in looking for ellipse around each point of the event and measure the longest arc which does not cross any region containing signals. If this arc is longer more than $\pi$, it is identified as a vertex (see Fig. 3a). 


\subsection{Fit of the 2D vertex}

The next step is to map the charge distributionl around the vertex in polar coordinates. Then only the regions with continuous signals from the vertex is selected. Most of the signal from the $\delta$-electron is removed. The peaks of the distribution obtained this way indicate the direction of the tracks originating from the vertex (Figure $3 b$ ).

\subsection{D vertex matching for 3D reconstruction}

In each plan $\mathrm{X}(\mathrm{t})$ and $\mathrm{Y}(\mathrm{t})$, the signal contained in a region around the track direction is associated with each of the two tracks (Figure 3c). Then, by comparing the charges profiles of the tracks as a function of the temporal coordinate $(t \propto Z)$, the $\mathrm{XZ}$ projection of a vertex is associated with its YZ projection (Figure 3d), so that finally the 3D vertex of the two tracks corresponding to the electron and the positron are recovered allowing to measure the incident photon direction and its polarisation.

\section{Results and discussion}

By reconstructing the directions coming from the vertices, the data analysis was performed to extract the polarisation asymetry and the angular resolution in function of the photon energy and compared to the simulation. Due to systematic biases, Figure $4 \mathrm{a}$ shows, from $11.8 \mathrm{MeV}$ data, that the distribution of $\phi_{ \pm}$of unpolarised photon $(\mathrm{P}=0)$ have a sinusoidal shape similar to the polarised $(\mathrm{P}=100 \%)$ distribution. By dividing the azimutal angle distribution for polarised photons by the unpolarised photons distribution extracted from the data, the systematics are cancelled and an asymmetry of polarisation A can be measured and compared to simulation (Figure 4b). For $11.8 \mathrm{MeV}, \mathrm{A}=13.0 \pm 0.8 \%$.

Data have been taken up to $74 \mathrm{MeV}$, but only unpolarised data up to $20 \mathrm{MeV}$ were taken. From those data, the asymetry of polarisation coefficients have been extracted and plotted on Figure 5a in function of the photon energy. Severals ratio of angle azimutal distibutions have been compared:

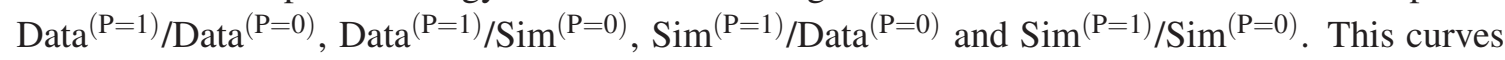
illustrate the ability of the HARPO prototype to measure polarised $\gamma$-ray photons from $1 \mathrm{MeV}$ to $20 \mathrm{MeV}$. But, the difference between the blue and the red curves shows also that our simulation is not complete for polarised photons $(\mathrm{P}=1)$.

The angular resolutions (68\% containment angle of the residual distribution of the photon direction) have been measured and is shown on Figure $5 \mathrm{~b}$ and compared to our simulation and to Fermi. HARPO demonstrator already achieved a two times better angular resolution than the Fermi-LAT. The angular resolution in high energy region $(>50 \mathrm{MeV})$ is expected to be improved on small vertex opening angle and where the multiple scattering is less important. Indeed, in a larger detector it will be possible to reconstruct the vertex by using the hit-points that are far from it.

\section{Large prototype for balloon flight}

The next step before a space flight telescope is to design and characterize a larger prototype during a stratopheric balloon flight. This prototype model so-called Self-Triggered Time projection chamber as a Gamma-ray Telescope (ST3G) is a cubic set of $4 \times 4 \times 4$ modules identical to 
HARPO, that is a $1.2 \mathrm{~m}^{3}$ sensitive gas volume. It will contain a argon-based mixture at a pressure of $\mathrm{P}=2$ bar. The anode strips will be enlarged to $4 \mathrm{~mm}$, which should make sure that the single-point space resolution $\sigma$ stays smaller than $1 \mathrm{~mm}$ over the whole drift length. In ST3G, most conversion events will have at least one track that crosses at least one amplification plane. For these, the timing precision with respect to the readout electronics clock has been measured to be $70 \mathrm{~ns}$ (RMS).

For a balloon flight or in space, the trigger will have to discriminate pair production events coming from $\gamma$-ray conversion from background events (traversing tracks events from cosmic rays). During the beam test, the trigger was built using the scintilators signals. But, thanks to the electronics ASTRE designed for TPC readout, the trigger will have to be build from detector signals [?]. In order to confirm such purpose, preliminary studies have been made by using a simple Monte Carlo simulation shown on Figure 6 that creates simplified detector and random straight tracks. Since no realistic detector response is implemented, the multiplicity signals of the chip mimics simply by using a segment length of projected tracks in a detector volume. This corresponds to the case of detectors with infinite pitch-size strips, infinite sampling frequency, and without signal smearing effect, e.g. diffusion.

The 3D signals are projected into three 2D-signals along X-, Y-, Z-axis in this study. The projections consist of cube-projections, e.g. $4 \times 4 . \mathrm{X}^{\prime}$ and $\mathrm{Y}^{\prime}$ are defined in each projection as horizontal axis and vertical axis respectively, and $\mathrm{Z}^{\prime}$ as the projecting direction. The projection simply adds, along $\mathrm{Z}^{\prime}$, the multiplicity signals in $\mathrm{X}^{\prime}$ and $\mathrm{Y}^{\prime}$ individually.

We focus on the fact that a single straight track event can be identified when each cubeprojection has an identical track-slope, which is computed as the ratio of the projected track length in $\mathrm{X}^{\prime}$ and the one in $\mathrm{Y}^{\prime}$. In practice multiplicity signals can be used instead of the projected track length taking into account the fact that the multiplicities are discrete values while the projected track lengths are continuous values. A parameter as a tolerance to cope with such a difference has been introduced. The tolerance parameter should be optimised depending on detector parameter such as strip-pitch, sampling frequency, diffusion, etc.

The primitive idea explained above have been tested with a limited number of events (4000 pair-track events as signals and 4000 single-track events as background). The preliminary result is encouraging us to proceed more detailed studies with a more sophisticated simulation.

\section{Conclusions}

Using a TPC demonstrator we were able to take polarised and unpolarised $\gamma$-ray data between 1.7 MeV and 74.3 MeV. The data analysis compared with our simulation shown that the performance of such detector (angular resolution: $8.8^{\circ} @ 11.7 \mathrm{MeV}$, polarisation asymmetry measurement capability: 13\%@11.7 MeV) have validated up to $20 \mathrm{MeV}$.

The design of a larger detector is on study and is an inevitable path to the space-flight experiment long-awaited by the astrophysical community in order to fill up the gap between $1 \mathrm{MeV}$, and $100 \mathrm{MeV}$.

This work was funded by the French National Research Agency (ANR-13-BS05-0002) and was performed by using NewSUBARU-GACKO (Gamma Collaboration Hutch of Konan University). 


\section{References}

[1] H. Zhang and M. Böttcher, X-ray and gamma-ray polarisation!! in leptonic and hadronic jet models of blazars, A. P. K. 774, 18 (2013).

[2] D. Bernard, TPC in gamma-ray astronomy above pair-creation threshold, Nucl. Instrum. Meth. A, vol. 701, p. 225, 2013.

[3] D. Calvet, A Versatile Readout System for Small to Medium Scale Gaseous and Silicon Detectors, IEEE Trans. Nucl. Sci. 61 (2014) 675.

[4] F. Sauli, GEM: A new concept for electron amplification in gas detectors, Nucl. Instrum. Meth. A 386, 531, 1997.

[5] Y. Giomataris et al., MICROMEGAS: A high-granularity position-sensitive gaseous detector for high particle-flux environments, Nucl. Instrum. Meth. A 376, 29, 1996.

[6] I. Giomataris et al., Micromegas in a bulk, Nucl. Instrum. Meth. A 560, 405, 2006.

[7] P. Gros et al., HARPO - TPC for High Energy Astrophysics and Polarimetry from the MeV to the GeV, Proceedings of Science, vol. TIPP2014, p. 133, 2014.

[8] A. Delbart et al., HARPO, TPC as a gamma telescope and polarimeter: First measurement in a polarised photon beam between 1.7 and $74 \mathrm{MeV}$, Proceedings of Science, vol. ICRC2015, p. 1016, 2015

[9] Y. Geerebaert et al., Electronics for HARPO: Design, Development and Validation of Electronics for a High Performance Polarised-Gamma-Ray Detector IEEE-RT, 2016

[10] R. Yonamine et al. A comprehensive analysis of polarised $\gamma$-ray beam data with a HARPO demonstrator, Procceding submitted from the TIPP17 Conference.

[11] P. Gros et al. Performance measurement of HARPO: a Time Projection Chamber as a gamma-ray telescope and polarimeter, arXiv:1706.06483 (2017)

[12] P. Gros et al. First measurement of the polarisation asymmetry of a gamma-ray beam between 1.7 to $74 \mathrm{MeV}$ with the HARPO TPC, Proc. SPIE Int. Soc. Opt. Eng. 9905, 99052R (2016) 


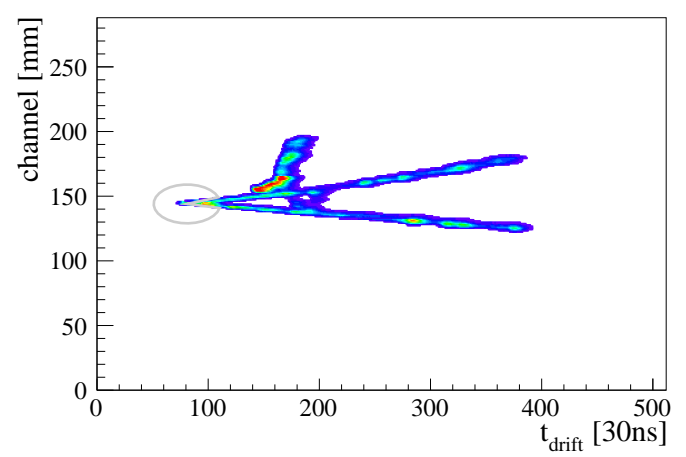

(a) 2D vertex finder procedure: circles are drawn around signal regions. If the longest arc without signal is larger than half an ellipse, the region is considered as a vertex candidate.
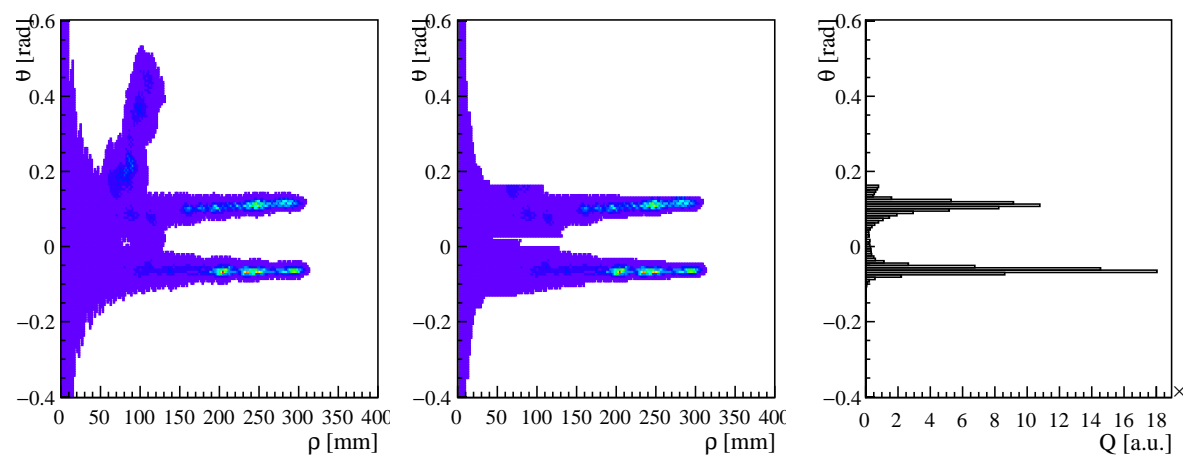

(b) 2D vertex fitting procedure: Left: polar distribution of charge around the vertex candidate. Middle: selection of regionsnearly continuous signal from the vertex. Right: angular distribution with two clear peaks corresponding to the two associated tracks.
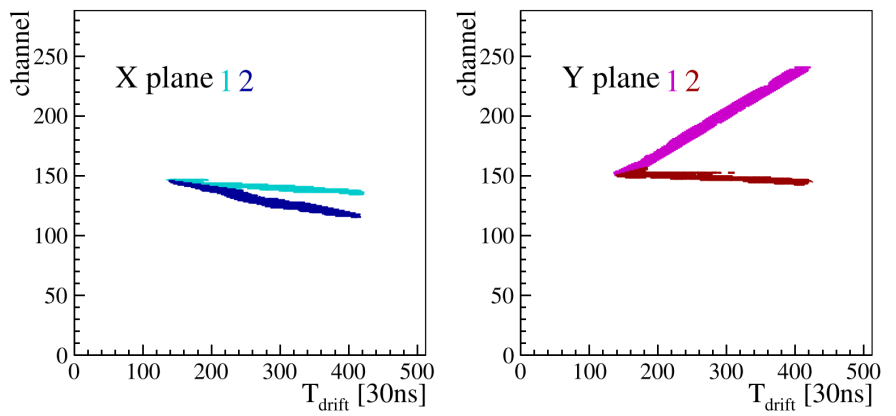

(c) Matching procedure: definition of the region associated to each of the two particles in the vertex
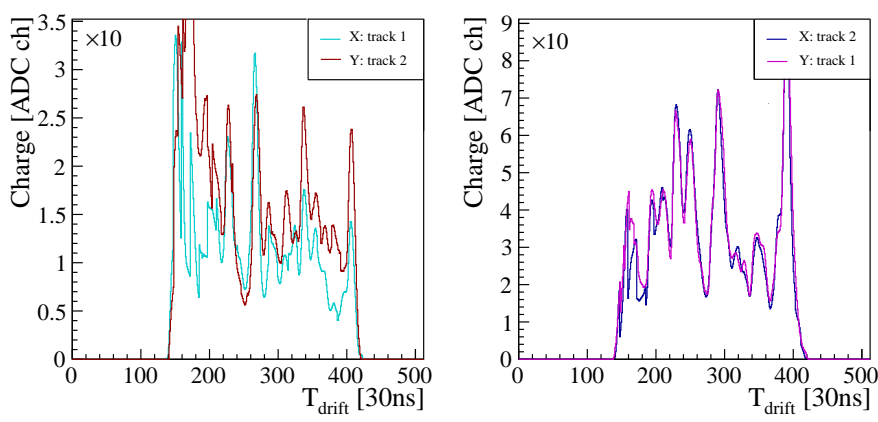

(d) Comparison of the charge profile (in $Z$ direction) of $X-Y$ track combinations.

Figure 3: Procedures to recontruct the vertex. 


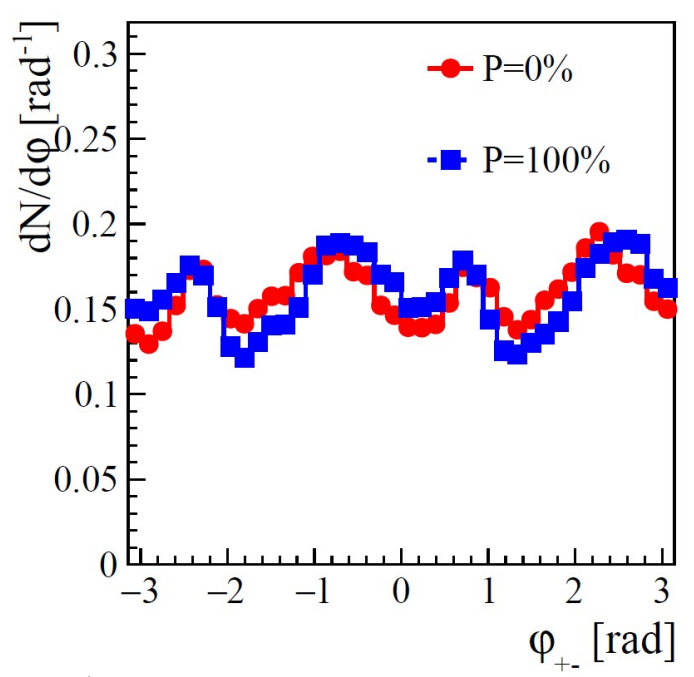

a)

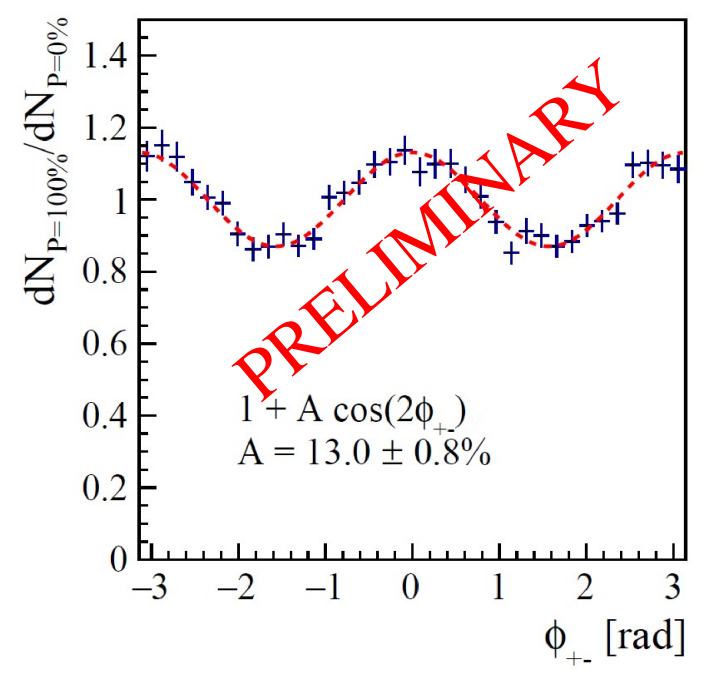

b)

Figure 4: polarisation plots from $11.8 \mathrm{MeV}$ photons with HARPO TPC oriented at $0^{\circ}$ around the beam axis: a) Distribution of $\phi_{ \pm}$extracted from data of polarised and unpolarised beam are shown with the blue squares and the red dots respectively. b) Ratio of the azimuthal angle distributions for polarised $(\mathrm{P}=100 \%)$ and unpolarised $(\mathrm{P}=0 \%)$. The systematic bias is cancelled by dividing the azimuthal angle distribution for polarised photons by the distribution for unpolarised photons from experimental data.

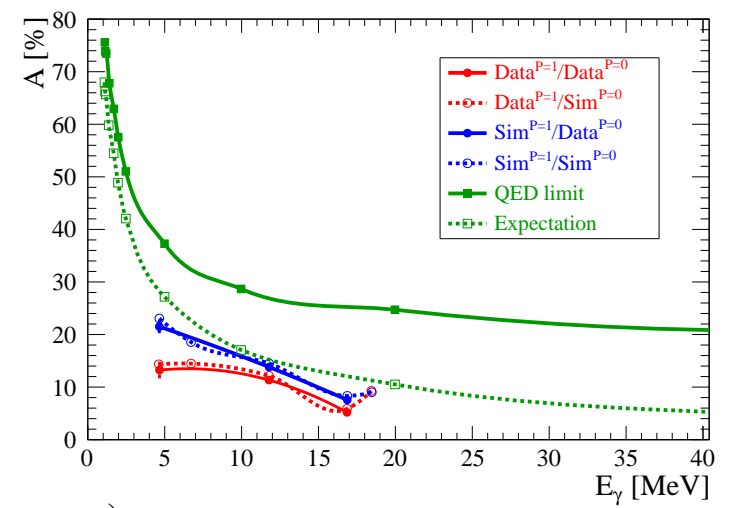

a)

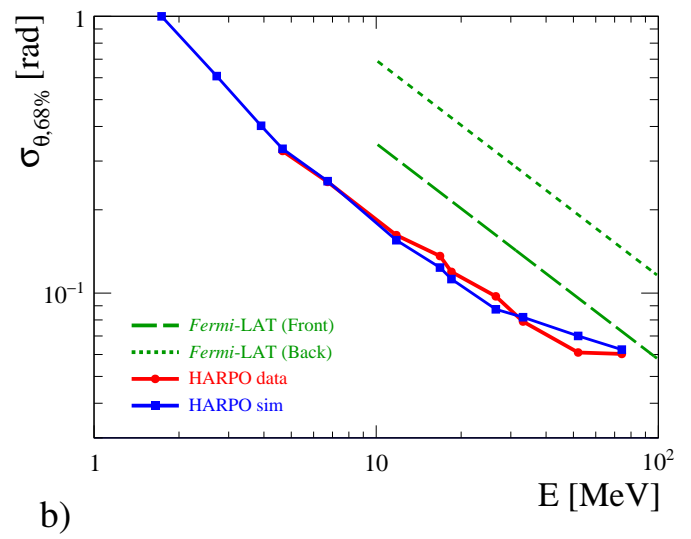

b)

Figure 5: Summary plots of the HARPO results. a) polarisation asymmetry measurment of $\gamma$-ray energy between 1 and $20 \mathrm{MeV}$. b) Comparison of the angular resolution between simulation and data. 
a) Traversing track

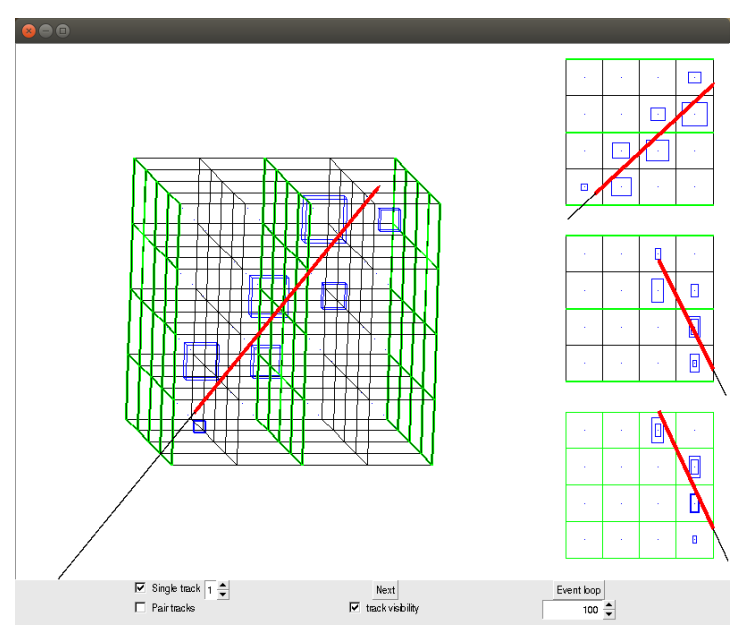

b) Pair production

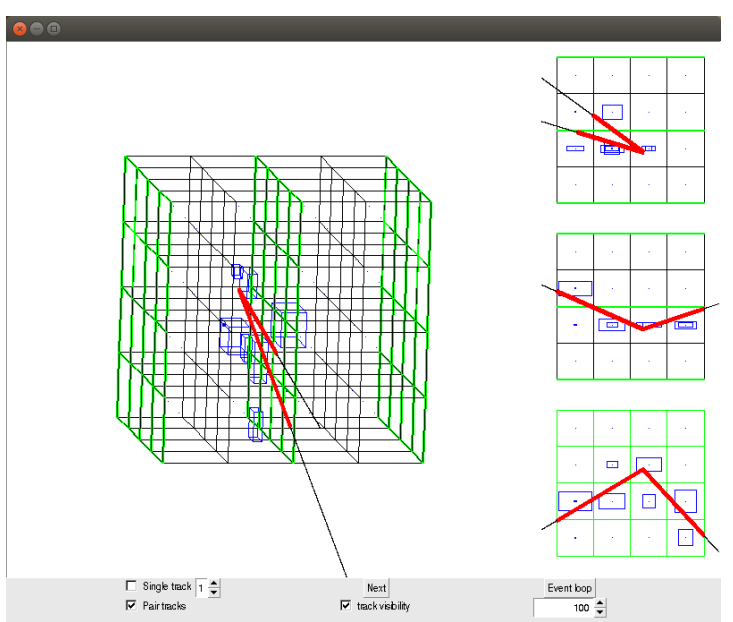

Figure 6: Toy model Monte Carlo to study trigger for the ST3G prototype. a) Traversing track from cosmic ray. b) Tracks from $\gamma$-ray pair conversion. 